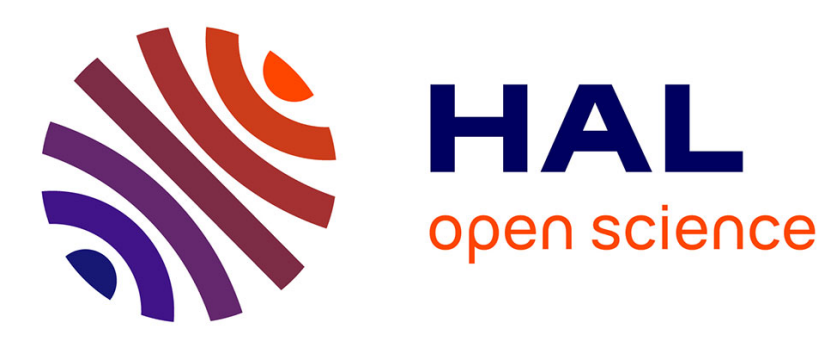

\title{
Application de la théorie de Lancaster à la consommation de poulet de chair au Cameroun
}

Catherine Laroche-Dupraz, Cyprien Awono, Dominique Vermersch

\section{To cite this version:}

Catherine Laroche-Dupraz, Cyprien Awono, Dominique Vermersch. Application de la théorie de Lancaster à la consommation de poulet de chair au Cameroun. Revue d'Etudes en Agriculture et Environnement - Review of agricultural and environmental studies, 2008, 86, pp.79-98. hal-01201166

\section{HAL Id: hal-01201166 https://hal.science/hal-01201166}

Submitted on 17 Sep 2015

HAL is a multi-disciplinary open access archive for the deposit and dissemination of scientific research documents, whether they are published or not. The documents may come from teaching and research institutions in France or abroad, or from public or private research centers.
L'archive ouverte pluridisciplinaire $\mathbf{H A L}$, est destinée au dépôt et à la diffusion de documents scientifiques de niveau recherche, publiés ou non, émanant des établissements d'enseignement et de recherche français ou étrangers, des laboratoires publics ou privés. 


\title{
Application de la théorie de Lancaster à la consommation de poulet de chair au Cameroun
}

\author{
Catherine LAROCHE DUPRAZ*, Cyprien AWONO**, \\ Dominique VERMERSCH*** \\ * Agrocampus Rennes, UMR SMART, 65 rue de Saint-Brieuc, CS 84215, F-35042 Rennes cedex, \\ France \\ e-mail:laroche@agrocampus-rennes.fr \\ ** INRA, UMR 1302, Rennes \\ *** Agrocampus Rennes, Département ERG
}

Résumé - Depuis la fin des années 1990, la production camerounaise de poulet de chair est fortement concurrencée par les importations à bas prix de poulet congelé en provenance du Brésil ou d'Europe, entraînant une crise de la filière. Une enquête réalisée à Yaoundé a montré les différences d'attributs de consommation des poulets locaux versus importés. Sur cette base, cet article développe une approche originale des déterminants de la consommation urbaine de poulet au Cameroun en utilisant la "Characteristics" theory" de Lancaster (1966a). Appliqué au cas de la consommation de poulet au Cameroun, ce cadre d'analyse est utilisé pour représenter les effets de deux leviers d'action en faveur de la reconquête du marché intérieur du poulet local: l'adaptation de la filière avicole aux attentes des consommateurs et une campagne de sensibilisation ciblée. Les apports et limites de ce travail sont enfin discutés, afin d'en dégager des conclusions et perspectives.

Mots-clés : consommation, théorie du consommateur, Lancaster, poulet, Cameroun

\section{The Lancaster's theory applied to local chicken consumption in Cameroon}

Summary - Since the end of the 1990's imports of chicken from Brazil and Europe compete with local chicken production in Cameroon, leading to a severe crisis in the country's poultry production. This article analyses the determinants of chicken urban consumption in Cameroon.

According to Lancaster's characteristic theory (1966), goods are differentiated by attributes revealed via the consumption technology. Consumers choose the goods through utility maximisation with respect to the goods' consumption characteristics, given the budget constraint, the availability of the goods, and the relative prices of each competing good. Data collected in Yaoundé suggest that it is simplistic to consider chicken as a homogeneous good: imported and local chicken exbibit different levels of consumption characteristics, e.g. in terms of patriotism vs convenience. Using Lancaster's approach in that case let us show the effects of an adaptation of local poultry production to new consumer's expectations vs an advertising campaign for local chicken.

Keywords: consumption, consumer theory, Lancaster, chicken, Cameroon

Descripteurs JEL : D12, D13, Q17, Q18

Nous remercions les deux lecteurs anonymes qui, par leurs remarques et suggestions, ont contribué à l'amélioration de cet article. 


\section{Introduction}

En initiant la «Characteristics' theory ", Lancaster (1966a) a permis un développement original de la théorie microéconomique du consommateur. Cet auteur y suggère en effet que les biens sont différenciés les uns des autres suivant un certain nombre d'attributs de consommation, intégrés par le consommateur dans une technologie de production domestique (bousebold production technology). Les choix des biens (ou outputs) par les consommateurs sont donc déterminés par la maximisation de l'utilité retirée des attributs de consommation (ou inputs) des biens, sous contrainte de revenu et de disponibilité des biens sur le marché, et compte tenu des prix relatifs des différents produits en concurrence.

Au Cameroun, comme dans plusieurs pays d'Afrique, la production locale de poulet de chair est, depuis la fin des années 1990, fortement concurrencée par les importations à bas prix de poulet congelés en provenance du Brésil ou d'Europe. Ce mouvement s'est accompagné d'une forte baisse des prix des viandes de poulet, occasionnant une grave crise de la filière avicole, dénoncée par les filières locales et les organisations non gouvernementales (ONG) internationales. Or, une analyse empirique de la consommation urbaine de poulets de chair au Cameroun indique que le poulet n'est pas un produit homogène, dans la mesure où les découpes importées de poulet congelé et le poulet de chair local qu'elles concurrencent présentent des attributs de qualité distincts.

Cet article vise à développer une analyse des déterminants de la consommation de poulet en milieu urbain au Cameroun, tenant compte des différents attributs de qualité des poulets observés en milieu urbain dans le cadre d'une enquête de consommation réalisée à Yaoundé en 2005. L'enjeu est de mettre en évidence d'éventuels leviers d'action possible permettant au poulet de chair local de reconquérir des parts de marché intérieur perdues.

La section 2 explique en quoi l'application de la théorie de Lancaster au cas de la consommation de poulet au Cameroun constitue un exercice qui vient compléter les travaux de recherches existants et qui portent sur la différenciation des produits alimentaires en Afrique sub-saharienne. La section 3 présente le cadre d'analyse de base appliqué au cas de la consommation de poulet au Cameroun; ce cadre est ensuite utilisé pour représenter les effets d'actions visant la reconquête, par le poulet local, des parts de marché perdues. Ainsi sont analysés les effets d'une adaptation de la filière avicole aux attentes des consommateurs, d'une part, et d'une campagne de sensibilisation, d'autre part. Dans la section 4, nous discutons les apports et limites de ce travail, afin d'en dégager des conclusions et perspectives.

\section{Contexte et cadre théorique de l'étude}

\subsection{La prise en compte de la différenciation des produits dans l'analyse de l'évolution de la consommation alimentaire dans les pays en développement}

L'accélération de l'urbanisation en Afrique sub-saharienne a des répercussions importantes sur les modes d'alimentation de la population. L'une d'entre elles est le développement d'opportunités, pour les produits agricoles élaborés en milieu rural, de 
conquête ou reconquête des marchés urbains. Toutefois, comme le soulignent Cheyns et Bricas (2003), cette évolution s'accompagne généralement de l'accroissement des importations de produits alimentaires en provenance du marché international, visant à assurer la sécurité alimentaire des grandes villes, ce qui participe à la concurrence des produits locaux par des produits importés à bas prix.

L'approche économique du marché d'un produit local concurrencé par les importations conduit classiquement à l'élaboration d'un modèle d'équilibre partiel. Sous l'hypothèse de l'homogénéité du bien considéré quelle que soit sa provenance, les prix du produit local versus importé constituent les déterminants essentiels de la répartition des parts du marché entre origines. Dans le cas d'une volonté politique de modifier cette répartition, par exemple pour limiter la part des importations dans la consommation totale, ce type de modèle permet de mesurer les effets des instruments de protection aux frontières sur les agents économiques du pays importateur.

L'hypothèse d'homogénéité du produit pose néanmoins problème, et l'analyse sur le terrain permet de se rendre compte que les consommateurs des pays en développement (PED) ont, comme dans les pays développés, des attentes multiples en termes de consommations alimentaires. Cheyns et Bricas (2003) montrent, par exemple, l'attention portée par les consommateurs de Ouagadougou (Burkina Faso) à différents indicateurs de qualité sanitaire des aliments ou de conformité à des normes de fabrication dans le cas des céréales, de la viande et du soumbala. Sanogo et Masters (2002) montrent la disponibilité à payer des mères de jeunes enfants pour une certification crédible de la qualité nutritionnelle des compléments alimentaires infantiles. Or les attributs de qualité des produits peuvent varier notamment en fonction de l'origine du produit. Ainsi d'après Lançon et Benz (2007), les consommateurs ouest africains sont prêts à payer plus cher le riz importé, mieux lavé et mieux emballé que le riz local; Dury et al. (2007) indiquent que les consommateurs maliens, malgré leur pauvreté, sont prêts à payer plus cher le fonio importé de Guinée, car considéré de meilleure qualité (plus clair, plus propre et mieux décortiqué) que le fonio local.

Ces études de cas, parmi d'autres, montrent que dans le cas où les modifications alimentaires favorisent la consommation des produits importés, du fait d'attributs différenciés, une politique douanière seule s'avère insuffisante et inadaptée pour protéger le marché domestique de la concurrence des produits importés. En effet, la définition de stratégies politiques ou industrielles visant à améliorer la qualité des produits domestiques (et leur certification) nécessite au préalable une connaissance précise de l'attente des consommateurs en matière de qualité, et impose donc de prendre en compte la différenciation des produits en concurrence.

Lancaster (1966a, 1966b) a développé une approche de la consommation, par analogie à la théorie de la production, qui a complété substantiellement la théorie standard. Selon cette approche, l'utilité du consommateur ne provient pas directement des biens consommés eux-mêmes, mais est issue d'une technologie de production domestique intégrant comme inputs une combinaison (un vecteur) d'attributs (également appelés caractéristiques).

De nombreux travaux se sont appuyés sur l'approche de Lancaster pour chercher à mesurer le consentement à payer pour les différents attributs de consommation des biens, 
en particulier lorsque la qualité n'est pas directement valorisée par le marché (Coestier et Marette, 2004). Dans le cas des biens alimentaires en Afrique de l'Ouest, Jabbar (1998), sur le marché des chèvres et des moutons au Nigéria, et Dalton (2004) sur la consommation de riz en Afrique de l'Ouest, utilisent des régressions hédoniques pour révéler les attributs des produits qui déterminent les préférences des consommateurs. Sanogo et Masters (2002) évaluent la demande pour la certification des aliments infantiles au Mali au moyen de méthodes relevant de l'économie expérimentale.

De tels travaux permettent de connaître la valeur des attributs accordée par les consommateurs. En revanche, ils n'éclairent pas toujours le décideur public sur la nature des effets, pour les consommateurs, de la mise en œuvre d'une politique destinée à favoriser un produit plutôt que son concurrent. Or Lancaster, en analysant les effets d'une modification des prix relatifs, ou le rôle de l'information relative aux produits en concurrence, avait initié une réflexion dans ce sens. Le présent article vise à revenir au cadre originel développé par Lancaster et à l'appliquer au cas de la consommation camerounaise de poulet, afin d'éclairer sur les leviers d'action possibles en faveur de la consommation de poulet local, et évaluer leurs effets sur les consommateurs.

\subsection{Bref rappel du cadre théorique d'analyse de la consommation de Lancaster}

L'approche de Lancaster permet de rendre compte de la possibilité pour un bien de présenter un vecteur d'attributs distincts, eux-mêmes pouvant éventuellement être présents et combinés à d'autres attributs en quantité variable et issus de la consommation d'autres biens.

Lancaster prend l'exemple d'un consommateur dans une économie où il existe deux attributs, 1 et 2 , obtenus conjointement selon une combinaison propre à la consommation de chacun des trois biens, $\mathrm{A}, \mathrm{B}$ et $\mathrm{C}$, disponibles sur le marché. Tenant compte de la contrainte budgétaire du consommateur, du prix relatif des trois biens et des combinaisons (ou vecteurs) d'attributs $(1,2)$ propres à la consommation de chaque bien $\mathrm{A}, \mathrm{B}$ ou $\mathrm{C}$, Lancaster détermine graphiquement l'ensemble des consommations possibles et la frontière d'efficacité allocative des consommations, dans un plan ayant pour abscisse et ordonnée respectivement les quantités d'attributs 1 et 2 issus de la consommation des biens. Sur la frontière d'efficacité, le choix du point de consommation effective (panier de biens) correspond aux préférences du consommateur. Les variations du revenu du consommateur se traduisent par un élargissement versus un rétrécissement de la frontière. De même, une variation, par exemple à la hausse, du prix relatif d'un des trois biens déplace la frontière d'efficacité, et peut même aboutir à la substitution d'un bien à un autre, devenu trop cher.

Le développement de cette théorie permet à Lancaster de promouvoir toutes les actions en faveur d'une meilleure information des consommateurs quant aux attributs de consommation des différents biens. Ainsi les informations dispensées par les associations de consommateurs, les labels et autres étiquettes d'information sur la composition des produits, mais aussi sa propre éducation, doivent permettre au consommateur de choisir au mieux son panier de biens compte tenu de ses préférences et sa contrainte budgétaire. De plus, l'arrivée sur le marché d'un nouveau produit, innovant par la combinaison inédite de ses attributs, est facilement intégrée à ce schéma, ce qui permet de traiter de certaines formes de différenciation d'un produit. 


\subsection{L'offre de poulet au Cameroun en milieu urbain}

On distingue au Cameroun deux principales filières de production de poulets, auxquelles s'ajoute l'importation de morceaux de poulets depuis l'étranger.

Le poulet «villageois » est produit dans de petites unités en milieu rural et en plein air. Il est principalement auto-consommé ou bien acheté au village par les consommateurs urbains, essentiellement à l'occasion de fêtes religieuses ou cérémonies traditionnelles. La portée symbolique de la consommation du poulet villageois est forte ; les autres types de poulets disponibles sur le marché camerounais se prêtent mal à ces utilisations spécifiques.

Le «poulet de chair local» est produit de façon rationalisée dans des élevages péri-urbains du Cameroun. Si la main-d'œuvre employée dans ces unités de production est locale, les poussins d'un jour, l'alimentation, les produits vétérinaires et autres intrants ainsi que la technologie de production, sont importés depuis l'Union européenne, la France et la Belgique en particulier (CCIMA, 2005). Les données concernant la production nationale different selon les sources, mais il semble qu'elle oscille entre 13000 et 30000 tonnes par an, en érosion constante depuis le milieu des années 1990 (ACDIC, 2005; Teleu Ngandeu et Ngatchou, 2006, FAOSTAT). Ce produit est destiné à la consommation urbaine de viande de poulet; il est vendu essentiellement entier et vivant sur les grands marchés urbains et périurbains du Cameroun.

Jusqu'en 1995, l'importation au Cameroun de viande de poulet depuis l'étranger était très limitée et réservée à des consommateurs aisés. En effet, le poulet importé était considéré comme un produit de luxe, car issu de technologies avancées « du Nord » (Communications personnelles des habitants de Yaoundé, Cameroon Tribune 20/08/ 2004). De plus, les droits de douanes élevés (20\%, auxquels s'ajoutent la TVA et la taxe phytosanitaire, $17,5 \%$ et $3 \%$ respectivement) appliqués aux importations de produits avicoles élevaient le prix de ces produits importés sur le marché camerounais à un niveau supérieur au prix du poulet local. A partir de 1995 et jusqu'en 2005, la volaille a été classée « bien de première nécessité » et, à ce titre, assujettie à un droit de douane réduit à $5 \%$, les autres taxes restant inchangées (Direction générale des douanes du Cameroun; CCIMA, 2005). La réduction des droits de douane appliqués aux poulets importés a entraîné une augmentation massive des importations de découpes de poulet en provenance d'Europe et du Brésil à bas prix. Ainsi les importations camerounaises de poulet sont progressivement passées de quelques centaines de tonnes en 1995 à près de 20000 tonnes en 2003, quantité dépassant très largement, sur toute la période, les quantités normalement autorisées à l'importation chaque année (ACDIC, 2004, d'après le ministère de l'Elevage, des Pêches et des Industries animales du Cameroun) ${ }^{1}$. Ces importations entrent en concurrence avec le poulet issu d'élevages rationalisés locaux et auraient entraîné, entre 1996 et 2003, la

\footnotetext{
${ }_{1}$ Plusieurs articles de presse font état de la large satisfaction des consommateurs, mais aussi de leur étonnement, suite à la rapide "démocratisation de la consommation de poulet ", entrainée par les importations massives de poulets, à la fin des années 1990 (voir, par exemple, Cameroon Tribune du 05/09/2003 et du 20/08/2004).
} 
fermeture de neuf élevages sur dix et la perte de 100000 emplois dans la filière avicole camerounaise selon l'Association civile de défense des consommateurs (Njonga, 2005).

Cette situation a généré une importante campagne de sensibilisation à la sauvegarde de la production locale de poulets, dénonçant les exportations massives et subventionnées de «bas morceaux » (voir par exemple la campagne d'information et de mobilisation d'Oxfam France - Agir ici, lancée en 2004).

Il semble indéniable que l'accroissement rapide des importations à bas prix de poulet sur le marché camerounais a entraîné une crise de la filière volaille locale. En effet, si la baisse générale des prix du poulet s'est traduite par une augmentation totale de la consommation de cette viande, devenue plus accessible aux consommateurs à faible pouvoir d'achat, cette évolution n'a pas profité à la filière avicole locale. Le poulet importé à bas prix s'est largement substitué au poulet de chair local dans la consommation, ce dernier résistant mal à la concurrence. En revanche, le prix du poulet villageois s'est maintenu à un niveau supérieur aux autres (cf. encadré 1).

Un examen approfondi de la consommation urbaine de poulet indique que les consommateurs considèrent différemment les trois principaux types de poulets présents sur le marché. Si tous les types de poulets procurent de la viande, source de calories et de protéines aux consommateurs, d'autres attributs de consommation les différencient à leurs yeux.

\subsection{Attributs de consommation des différents types de poulet disponibles sur le marché et approche de la technologie de leur consommation}

Afin d'analyser plus finement les déterminants de la consommation urbaine de poulet au Cameroun, une enquête a été réalisée à Yaoundé entre mai et juillet 2005 (Awono et al., 2005). Cette enquête visait à identifier les modes de consommation des différents types de poulet. La ville de Yaoundé, qui compte environ un million et demi d'habitants, a été choisie, car elle est la capitale administrative du Cameroun et constitue un centre de consommation important.

La méthode non probabiliste des quotas a permis de prélever, parmi les personnes enquêtées, des échantillons représentatifs de 180 ménages, 180 consommateurs hors foyer, 60 restaurateurs et 45 vendeurs de poulet. Ces échantillons présentent une distribution de certains caractères ${ }^{2}$ statistiquement représentative de la population dans laquelle ils sont prélevés. La méthode des quotas a été couplée à la méthode des itinéraires qui permet de contrôler les enquêteurs, afin d'éviter les biais dans la collecte des données.

L'analyse des résultats d'enquêtes permet de dégager quelques éléments de caractérisation de la demande de viande de poulet en termes d'échelle de préférences des consommateurs, de déterminants de la qualité, du degré de substituabilité des

\footnotetext{
2 Par exemple, pour les ménages enquêtés : poids démographique des sites d'enquêtes, taille du ménage, répartition par sexe des enquêtés, catégories socioprofessionnelles, personne qui décide de l'achat de la nourriture au sein du ménages, type de logement, ...
} 


\section{Encadré 1. Les prix du poulet sur les marchés de Yaoundé, Cameroun}

Sur les marchés camerounais, les prix des produits ne sont pas affichés, car ils sont issus d'une négociation entre l'acheteur et le vendeur, et donc propres à chaque transaction. Ceci constitue un obstacle au relevé des prix d'un produit, d'autant qu'il est délicat, voire importun, de demander le prix d'un produit qui vient de faire l'objet d'une transaction. De plus, du fait de l'âpre négociation qu'il a eue avec le vendeur, l'acheteur pense le plus souvent avoir fait une bonne affaire. Aussi considère-t-il le prix d'achat du poulet comme un prix « d'associé » et peut être tenté de le majorer lorsqu'on lui demande à quel prix il a acheté le poulet. Il est par ailleurs difficilement envisageable de connaitre le poids du poulet de chair local pour chaque transaction, ce dernier étant vendu vif, essentiellement au jugé. Toute tentative d'estimation du poids peut être très mal interprétée aussi bien par le vendeur que par le consommateur. Il devient par conséquent quasiment impossible de disposer du prix au kilo du poulet si on ne l'achète pas. En revanche, en ce qui concerne les découpes de poulet importé congelées, la négociation entre acheteur et vendeur porte sur le prix au kilo, les produits échangés étant pesés au moment de l'achat.

L'Institut national de la statistique (INS, 2004) fournit des moyennes annuelles des prix à la consommation du poulet de chair local, basées sur des relevés mensuels de prix du poulet vif tirés d'enquêtes auprès des ménages, ramené au kilogramme sur la base d'une estimation du poids moyen du poulet dont la méthode n'est pas précisée. Le prix au kilogramme indiqué entre 2001 et 2003 (années les plus récentes dont les données sont disponibles) se stabilise autour de $1030 \mathrm{~F} \mathrm{CFA} / \mathrm{kg}$. Sur cette même période, le prix moyen donné par l'INS pour le poulet importé est supérieur: $1549 \mathrm{~F} \mathrm{CFA} / \mathrm{kg}$. Mais ces chiffres sont contestés par une partie des professionnels du secteur avicole camerounais. Ainsi la Chambre de commerce et industrie des mines et de l'artisanat (CCIMA, 2005) estime que le prix du poulet de chair local a chuté entre 1997 et 2003, en passant de 1950 à 1745 F CFA/kg. Ces données sont reprises dans Cameroon Tribune (du 07/10/2005) et l'étude de Teleu-Ngandeu et Ngatchou (2006) qui précise que le poulet importé est moins cher à la consommation que le poulet de chair local. Les professionnels de l'INS questionnés sur ces prix admettent que leur méthode de relevé aboutit certainement à une sous-estimation des prix du poulet de chair local dans la mesure où les prix indiqués ne semblent pas toujours cohérents avec la réalité observée sur les marchés (Communications personnelles avec des professionnels de l'INS).

L'enquête d'Awono et al. (2005) portant sur l'évolution de la consommation urbaine de poulet de chair à Yaoundé entre mai et juillet 2005 permet d'apporter des compléments d'information sur les prix. Une première approche sur le terrain a montré une grande variabilité des prix des produits selon le moment de la vente, les clients bénéficient d'un meilleur prix à l'ouverture et à la fermeture du marché. De même, la période d'achat détermine le prix des produits. Ainsi en période de fêtes, l'augmentation de la demande importante entraîne les prix à la hausse. Enfin les prix pratiqués sont variables en fonction des lieux de vente. 
Réalisée en journée et hors période de fête particulière, l'enquête a consisté à interroger, d'une part, 180 ménages répartis dans les six arrondissements de Yaoundé, à leur domicile, et, d'autre part, 180 vendeurs de poulet répartis dans les principaux marchés de Yaoundé (Mfoundi, Mokolo, Etoudi, Nkol-Eton, Emombo, Essos, Royal hôtel, Mvog-Mbi, Melen). Ces enquêtes ont été accompagnées de pesées de 130 poulets de chair locaux, mis en vente sur les grands marchés de Yaoundé, au moyen d'une seule et même balance, en dehors de transaction particulière.

Les prix du poulet de chair local relevés auprès des vendeurs, hors de transaction précise, sont compris dans une fourchette de 1200 à $1833 \mathrm{~F} \mathrm{CFA} / \mathrm{kg}$.

Les ménages ont été interrogés sur le prix maximum auquel ils consentiraient pour l'achat (i) d'un poulet villageois vif, (ii) d'un poulet de chair local vif, et (iii) d'un kilo de découpes de poulet congelé importé. En moyenne sur l'ensemble des ménages enquêtés, les prix déclarés sont les suivants : $3532 \mathrm{~F} \mathrm{CFA}$ pour un poulet villageois, $3360 \mathrm{~F} \mathrm{CFA} \mathrm{pour} \mathrm{un} \mathrm{poulet} \mathrm{de} \mathrm{chair} \mathrm{local} \mathrm{(coût} \mathrm{du} \mathrm{vidage} \mathrm{et} \mathrm{plumage}$ compris), et $1369 \mathrm{~F} \mathrm{CFA} \mathrm{pour} \mathrm{un} \mathrm{kilogramme} \mathrm{de} \mathrm{découpes} \mathrm{de} \mathrm{poulet} \mathrm{importé.} \mathrm{On}$ note que les habitants du centre ville font état de consentements à payer plus élevés qu'ailleurs. En utilisant le résultat de nos pesées du poulet local vif sur les marchés de Yaoundé (i.e. 1,7 kg en moyenne pour un poulet), le prix du poulet de chair local ramené au kilogramme s'établit en moyenne à $1972 \mathrm{~F} \mathrm{CFA} / \mathrm{kg}$. Les prix déclarés par les ménages pour les trois types de poulet sont reportés dans la figure 1 .

Figure 1. Prix des différents types de poulet

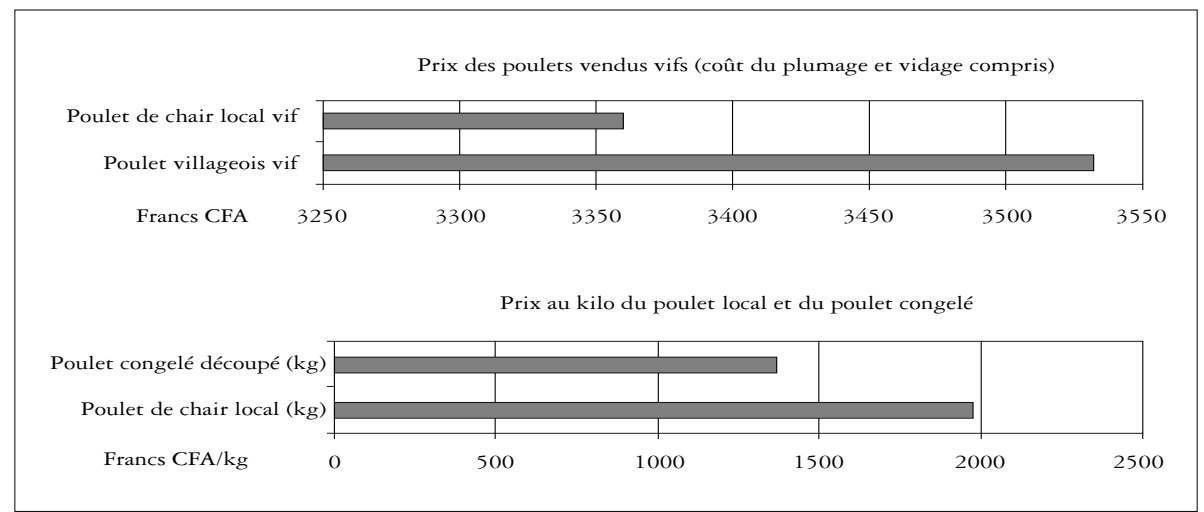

Source : Enquête réalisée à Yaoundé en 2005 auprès de 180 ménages, moyenne des déclarations des répondants.

Il ressort donc des déclarations des consommateurs au moment de l'enquête que, ramené au kilogramme, le prix du poulet de chair local est supérieur au prix d'un kilogramme de poulet importé congelé. Le prix indiqué par les consommateurs se situe en haut de la fourchette des prix déclarés par les vendeurs. 
différents produits avicoles disponibles sur les marchés camerounais, et de consentement à payer des consommateurs. Une pesée systématique des poulets vendus dans les principaux marchés de Yaoundé et de son agglomération a permis de recueillir des informations précieuses quant aux prix des différents types de poulet ramenés au $\mathrm{kg}$ de viande, tenant compte de la plumaison et l'éviscération pour le poulet de chair local.

Les résultats de cette enquête permettent de préciser les attributs de chaque type de poulet qui déterminent les choix des consommateurs urbains. Il en ressort notamment les éléments suivants :

- La consommation urbaine de poulet villageois est liée à des circonstances particulières (cérémonie traditionnelle ou fête religieuse) pour lesquelles les deux autres types de poulet disponibles sur le marché (poulet de chair local et découpes de poulet importé) ne sont pas adaptés.

- Le prix constitue le facteur principal dans le choix des consommateurs. Ainsi l'importation massive de morceaux de poulets vendus à bas prix vient-elle concurrencer directement la production locale de poulet de chair. Même si le prix au kilo du poulet de chair local reste proche du poulet importé, le fait que ce dernier soit essentiellement vendu en morceaux congelés, à la différence du poulet local vendu entier et vivant, fournit un avantage comparatif. En effet, les consommateurs à très faible pouvoir d'achat qui ne disposent pas d'une somme suffisante pour acheter un poulet local entier peuvent acheter à l'unité un petit nombre de morceaux de poulet importé.

- Toutefois, en dehors des considérations de prix, plus des deux tiers des répondants, qu'il s'agisse des ménages ou des consommateurs hors foyer, déclarent «préférer » le poulet de chair local aux découpes de poulet importé. Pour autant, les consommateurs semblent incapables de différencier, à l'aveugle, le goût des deux types de poulet. Ainsi cette préférence pour le produit national peut-elle sans doute davantage s'expliquer par d'autres facteurs: patriotisme économique, qualité sanitaire des poulets locaux perçue comme meilleure que les produits importés congelés... Ces autres facteurs étant par ailleurs fortement relayés par les actuelles campagnes de mobilisation contre les importations de poulet (Oxfam France - Agir ici, 2004).

- La vente à la part des découpes de poulet congelé est adaptée à la consommation des ménages à très faibles revenus : ceux-ci peuvent s'acheter des parts de poulet à l'unité alors qu'ils ne pourraient pas s'offrir un poulet entier: le caractère divisible du poulet rend celui-ci accessible. Autrement dit, l'attribut divisibilité du poulet importé fait émerger chez ces ménages pauvres un consentement à payer non nul pour avoir «du » poulet à consommer.

- Par ailleurs, pour les ménages aisés et pour les restaurateurs, le poulet vendu en découpes s'avère plus pratique que le poulet de chair local qu'il faut abattre, plumer, vider et découper avant préparation, et très adapté aux habitudes culinaires relatives à la préparation du poulet (poulets braisés, frits, sautés...).

Nous avons confronté la perception par les consommateurs des attributs de consommation et la nature effective de ces attributs dans la réalité, afin de faire ressortir les éléments qui semblent réellement déterminants dans les choix de consommation en milieu urbain. Étant donné le caractère tout à fait spécifique de la 
Tableau 1. Identification des attributs de consommation du poulet de chair local et des découpes de poulet congelées importées. Comparaison de la perception des consommateurs (telle qu'elle ressort des enquêtes menées) et d'autres éléments factuels à notre connaissance

\begin{tabular}{lllll}
\hline $\begin{array}{c}\text { Attributs de consommation } \\
\text { identifiés }\end{array}$ & \multicolumn{1}{c}{$\begin{array}{c}\text { Poulet de chair local } \\
\text { vendu entier et vif }\end{array}$} & $\begin{array}{c}>\text { ou } \\
(1)\end{array}$ & $\begin{array}{l}\text { Découpes de poulet } \\
\text { importées congelées }\end{array}$ \\
\hline $\begin{array}{l}\text { Qualité } \\
\text { gustative }\end{array}$ & $\begin{array}{l}\text { Perception } \\
\text { par le } \\
\text { consommateur }\end{array}$ & $\begin{array}{l}66 \% \text { des consommateurs de Yaoundé } \\
\text { déclarent préférer le poulet de chair } \\
\text { local au poulet congelé importé } \\
\text { (Awono et al., 2005). }\end{array}$ & $\begin{array}{l}> \\
\text { versatilité }\end{array}$ & $\begin{array}{l}\text { Avant les importations } \\
\text { massives de découpes de } \\
\text { poulet, les produits avicoles }\end{array}$ \\
& & & importés jouissaient d'une \\
& & & $\begin{array}{l}\text { bonne réputation, tant pour } \\
\text { leur goût que pour leur } \\
\text { qualité }\end{array}$ \\
\hline
\end{tabular}

\begin{tabular}{ll}
\hline Objectivement & Les tests en aveugle (notamment au $\quad>=<$ \\
& restaurant où le type de poulet servi versatilité \\
& n'est pas spécifié) ne permettent pas de \\
& confirmer la préférence affichée par les \\
& consommateurs. (Awono et al., 2005).
\end{tabular}

\begin{tabular}{|c|c|c|c|c|}
\hline $\begin{array}{l}\text { Qualité } \\
\text { sanitaire }\end{array}$ & $\begin{array}{l}\text { Perception } \\
\text { par le } \\
\text { consommateur }\end{array}$ & Versatilité des consommateurs. & $>$ & $\begin{array}{l}\text { Les campagnes actuelles de } \\
\text { mobilisation mettent } \\
\text { l'accent sur les fréquentes } \\
\text { ruptures de la chaîne du } \\
\text { froid de l'importation des } \\
\text { découpes jusqu'à la } \\
\text { consommation (voir, par } \\
\text { exemple, Oxfam France - } \\
\text { Agir ici, 2004). }\end{array}$ \\
\hline
\end{tabular}

Objectivement Les conditions d'approvisionnement et $>=<$ ?

de conservation des intrants et produits vétérinaires utilisés dans les élevages locaux ainsi que les conditions d'élevage, d'abattage et de conservation ne garantissent pas une qualité sanitaire irréprochable (2).

\begin{tabular}{|c|c|c|c|c|}
\hline \multirow[t]{2}{*}{$\begin{array}{l}\text { Intérêt } \\
\text { patriotique }\end{array}$} & $\begin{array}{l}\text { Perception } \\
\text { par le } \\
\text { consommateur }\end{array}$ & $\begin{array}{l}\text { La consommation de poulet de chair } \\
\text { local favorise le maintien des élevages } \\
\text { péri-urbains et donc le développement } \\
\text { économique du secteur avicole local. }\end{array}$ & $>$ & $\begin{array}{l}\text { Les importations de poulet } \\
\text { concurrencent la filière } \\
\text { avicole locale. }\end{array}$ \\
\hline & Objectivement & & $>$ & \\
\hline \multirow[t]{2}{*}{ Divisibilité } & $\begin{array}{l}\text { Perception } \\
\text { par le } \\
\text { consommateur }\end{array}$ & $\begin{array}{l}\text { Le poulet de chair local est vendu } \\
\text { entier, vif. Il peut toutefois } \\
\text { éventuellement être abattu, plumé et } \\
\text { découpé sur place par un plumeur } \\
\text { (contre rémunération modique du } \\
\text { service). }\end{array}$ & $<$ & $\begin{array}{l}\text { Le poulet importé est vendu } \\
\text { congelé, le plus souvent } \\
\text { morceau par morceau } \\
\text { (cuisse, aileron, gésier, etc.). }\end{array}$ \\
\hline & Objectivement & & $<$ & \\
\hline
\end{tabular}

(1) Le signe > signifie que l'attribut considéré est plus abondant dans le poulet de chair que dans les découpes de poulet congelées importées. Le signe < indique l'inverse.

(2) Communication personnelle des responsables du MINEPIA (Directions des services vétérinaires et des productions animales) et de nombreux acteurs de la filière avicole locale (éleveurs, minotiers et associations des consommateurs). 
consommation du poulet villageois en milieu urbain, ce dernier est exclu de l'analyse, qui se limite donc à la comparaison du poulet de chair local, d'une part, aux découpes de poulet congelé importé, d'autre part.

Notons qu'il serait éventuellement possible de fondre en un seul et même attribut le patriotisme et le goût. En effet, le résultat des enquêtes réalisées en 2005 indique une préférence, en terme de goût, pour le poulet de chair local qui témoigne sans doute davantage d'une perception dictée par l'origine du poulet que par une différence gustative significative, dans la mesure où cette préférence est démentie dans les faits lorsqu'on se retrouve en l'absence d'information sur l'origine du poulet. Les réponses aux enquêtes sur ce point pourraient se synthétiser dans une formule comme : "Cela me fait plaisir de consommer du poulet de chez nous ; d'ailleurs, il est meilleur ».

Par ailleurs, la notion de qualité sanitaire semble sujette à la versatilité des consommateurs, qui sont sensibles aux campagnes de mobilisation et d'information en cours. Or ces campagnes, qui visent à alerter des méfaits des importations de poulet, se fondent sur des considérations relatives au maintien de l'économie avicole locale (ce qui se confond avec l'attribut " patriotisme »), tout en mettant l'accent sur la faible qualité des produits avicoles importés.

La section 3 vise à mettre en application, à titre exploratoire, le cadre analytique originel de Lancaster, dans le cas de la consommation du poulet au Cameroun. Ceci nous permet de prendre en considération la différenciation des produits consommés, du fait des combinaisons distinctes d'attributs de consommation des différents types de poulet en concurrence sur le marché camerounais.

\section{Utilisation du modèle de Lancaster pour la modélisation de la consommation urbaine de poulet au Cameroun}

L'utilisation de la théorie de Lancaster pour la modélisation de la consommation urbaine de poulet au Cameroun ne prétend évidemment pas représenter l'ensemble de la problématique de la concurrence, sur le marché camerounais, du poulet congelé importé et du poulet local. Elle vise néanmoins à aborder cette question par une entrée originale, celle de la technologie de production domestique mettant en valeur certains attributs de consommation inégalement présents dans les différents poulets en concurrence et traite, au moins partiellement, de la différenciation des produits. En dépassant l'analyse classique d'un marché en équilibre partiel, supposant l'homogénéité du produit, nous espérons dégager des pistes plus fines quant aux possibilités de protéger les intérêts du secteur avicole camerounais.

Afin d'utiliser le modèle de Lancaster de façon simple, via une première représentation graphique à deux dimensions, nous limiterons à deux le nombre d'attributs retenus dans un premier temps, en retenant les attributs qui semblent subjectivement et objectivement les moins ambigus. Le goût, par exemple, est difficilement objectivable et semble sujet à la versatilité des consommateurs, sensibles aux campagnes d'information en cours; il nous sera difficile de le retenir en première approche. En revanche, l'analyse du tableau 1 nous incite à retenir "l'intérêt patriotique » et «la divisibilité » dont les signes sont très clairs. Le cadre d'analyse 
graphique ainsi défini, nous chercherons dans un second temps à déterminer comment y intégrer les effets d'une campagne de mobilisation en faveur du poulet local, ou d'une modification de la technologie de production de la filière avicole locale, qui tienne compte de la perception des attributs du poulet par les consommateurs.

Notons que dans toute l'étude, nous restons sous l'hypothèse d'invariance de la forme des courbes d'indifférence des consommateurs: leur courbure reste en permanence inchangée. En effet, conformément à l'analyse de Lancaster, nous nous intéressons à la perception, par les consommateurs, du niveau des attributs recherchés des deux types de poulet en concurrence, compte tenu de la fonction d'utilité donnée des consommateurs. Le niveau réel et objectif (d'ailleurs assez difficilement mesurable dans le cas de l'attribut 1 par exemple) de ces attributs, dans un contexte d'évolution des préférences des consommateurs, compte moins. Ce choix semble raisonnable tant que l'on reste dans le court terme. Il est néanmoins concevable d'imaginer que les préférences des consommateurs évoluent à long terme, toutefois l'économiste est peu armé pour évaluer les modifications des courbes d'indifférence des consommateurs.

\subsection{Cadre d'analyse graphique}

On suppose que deux biens sont disponibles sur le marché camerounais du poulet : le poulet de chair local noté $A$ et les découpes de poulet congelé importées notées $B$. Chacun des deux produits $\mathrm{A}$ et $\mathrm{B}$ sont vecteurs de deux attributs de consommation : le "patriotisme» noté 1 et la «divisibilité " notée 2 . On suppose que l'utilité des consommateurs croît avec le niveau de chacun des attributs. On suppose également dans ce cadre que la courbure de la courbe d'iso-utilité est invariante.

La demi-droite [OA) indique l'ensemble des consommations possibles si la totalité du budget est consacrée à la consommation du poulet $\mathrm{A}$; la demi-droite $[\mathrm{OB}$ ) indique l'ensemble des consommations possibles si la totalité du budget est consacrée à la consommation du poulet $\mathrm{B}$.

On suppose une situation initiale où les prix des poulets $A$ et $B$ sont respectivement $\mathrm{p}_{\mathrm{A}}$ et $\mathrm{p}_{\mathrm{B}}$. Le segment de droite $[\mathrm{AB}]$ est la partie accessible de la droite de budget initiale. Il regroupe l'ensemble des points d'équilibre obtenus, lorsque les consommateurs dépensent l'intégralité de leur budget, soit en poulets $A$ (point $A$ ), soit en poulets $B$ (point $B$ ), soit en une combinaison des deux poulets (segment $] A B[$ ). Les points $\mathrm{A}$ et $\mathrm{B}$ indiquent clairement qu'à budget égal consacré à l'achat de poulet, la consommation exclusive de poulet $A$ procure des niveaux d'attributs 1 et $2\left(A^{1} ; A^{2}\right)$ très différents des niveaux $\left(\mathrm{B}^{1} ; \mathrm{B}^{2}\right)$ procurés par la consommation exclusive de poulet $\mathrm{B}$; le poulet local A étant plus riche en attribut 1 (le patriotisme), le poulet importé découpé congelé $\mathrm{B}$ étant plus riche en attribut 2 (divisibilité).

On note U la courbe d'iso-utilité atteinte à l'état initial. E représente le point de consommation efficace, qui maximise l'utilité des consommateurs compte tenu de leur contrainte budgétaire. La demi-droite $[\mathrm{OE})$ indique la répartition initiale des consommations entre les poulets $\mathrm{A}$ et $\mathrm{B}$ sous la contrainte du budget des consommateurs. Elle constitue l'axe de déplacement de la courbe U lorsque le budget des consommateurs s'accroît. 
Graphique 1. Représentation graphique de la détermination du panier de consommation en fonction des attributs de consommation de chaque type de poulet

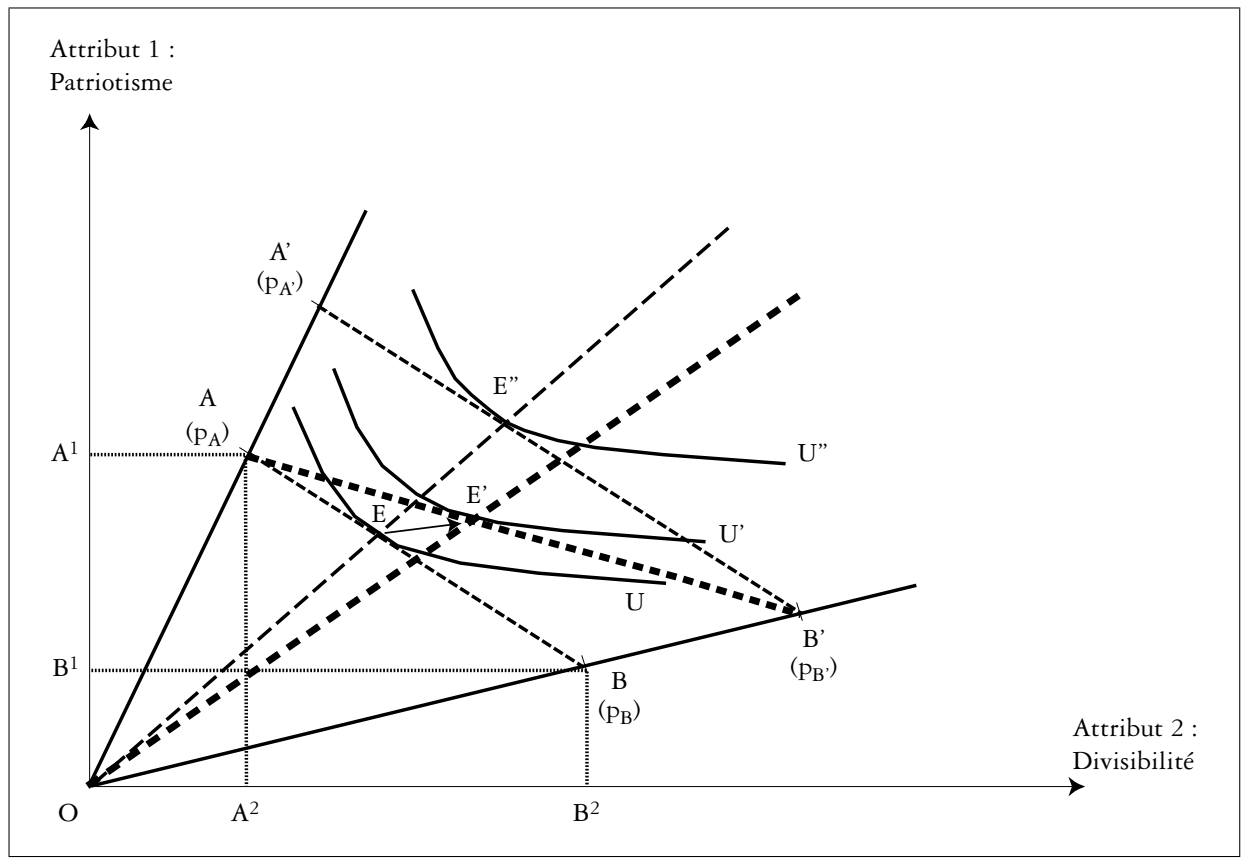

Supposons que le prix du poulet congelé $\mathrm{B}$ diminue de $\mathrm{pB}$ à $\mathrm{pB}$ ' suite, par exemple, à la réduction des barrières douanières, comme ce fut le cas à partir de 1995. Toutes choses égales par ailleurs, et en particulier sans modification de revenu des consommateurs, ce changement de prix entraîne un déplacement de la droite de budget de $[A B]$ à $[A B$ ']. De même, sans évolution de la forme des courbes d'iso-utilité, entre les deux situations, la variation à la baisse du prix du poulet $B$ va permettre d'atteindre un niveau d'utilité U' > U. L'équilibre efficace se déplace de E en E'. La pente de la nouvelle demi-droite [OE') indique naturellement une modification de la répartition de la consommation entre poulets en faveur d'une substitution du poulet $\mathrm{B}$ à une partie de la consommation de poulet A.

La question qui se pose à ce stade est de savoir si le poulet $\mathrm{A}$ peut retrouver sa part de marché initiale et de quelles manières. Dans un cadre d'analyse classique, la réponse évidente est que pour revenir à la situation initiale (demi-droite $[\mathrm{OE})$ ), deux voies sont possibles :

- soit réintroduire la protection initiale à l'entrée du poulet $B$, permettant un retour au point d'équilibre $\mathrm{E}$,

- soit abaisser suffisamment le prix du poulet $\mathrm{A}$ de façon à déplacer l'équilibre de E' vers E”, situé sur la demi-droite [OE). Le niveau d'utilité atteint U” > U'.

Ces éléments de réponse ignorent toutefois d'éventuelles autres voies à explorer dès lors que l'on prend en compte les attributs de consommation des deux poulets en concurrence sur le marché. 


\subsection{Les effets d'une adaptation de la technologie de production avicole locale aux attentes des consommateurs}

L'analyse de la consommation de poulet au Cameroun réalisée par le biais d'enquêtes a mis en évidence que si le prix relatif des deux types de poulet en concurrence constituait le principal déterminant du choix de consommer du poulet local versus des découpes de poulet importé, d'autres éléments étaient pris en considération par les consommateurs. En particulier la divisibilité des découpes de poulet congelé joue en faveur d'une plus grande substitution du poulet importé au poulet local, tandis que la mise en avant du « patriotisme » viendrait modérer cette tendance (cf. tableau 1).

Ces informations nous amènent, compte tenu de notre cadre d'analyse, à proposer une troisième voie permettant au poulet local de retrouver sa part de marché d'origine. Ainsi, le développement d'une filière locale d'abattage, plumage et découpage du poulet local permettrait de proposer sur le marché des découpes de poulet local. Le graphique 2 met en évidence dans quelle mesure cette troisième voie pourrait se révéler intéressante pour la filière avicole locale.

On part d'une situation initiale d'équilibre E' obtenue à l'issue de l'ouverture aux importations de découpes de poulet congelé. Le niveau d'utilité atteint est U'; la droite de budget passe par [AB'].

On suppose que se développe un changement technologique de la filière avicole locale: le poulet local est maintenant vendu abattu, plumé et découpé. Pour commencer, on pose l'hypothèse (peu vraisemblable) que ce changement technologique

Graphique 2. Effets d'une adaptation ad boc de la filière avicole camerounaise améliorant la divisibilité (attribut 2) du poulet local (A)

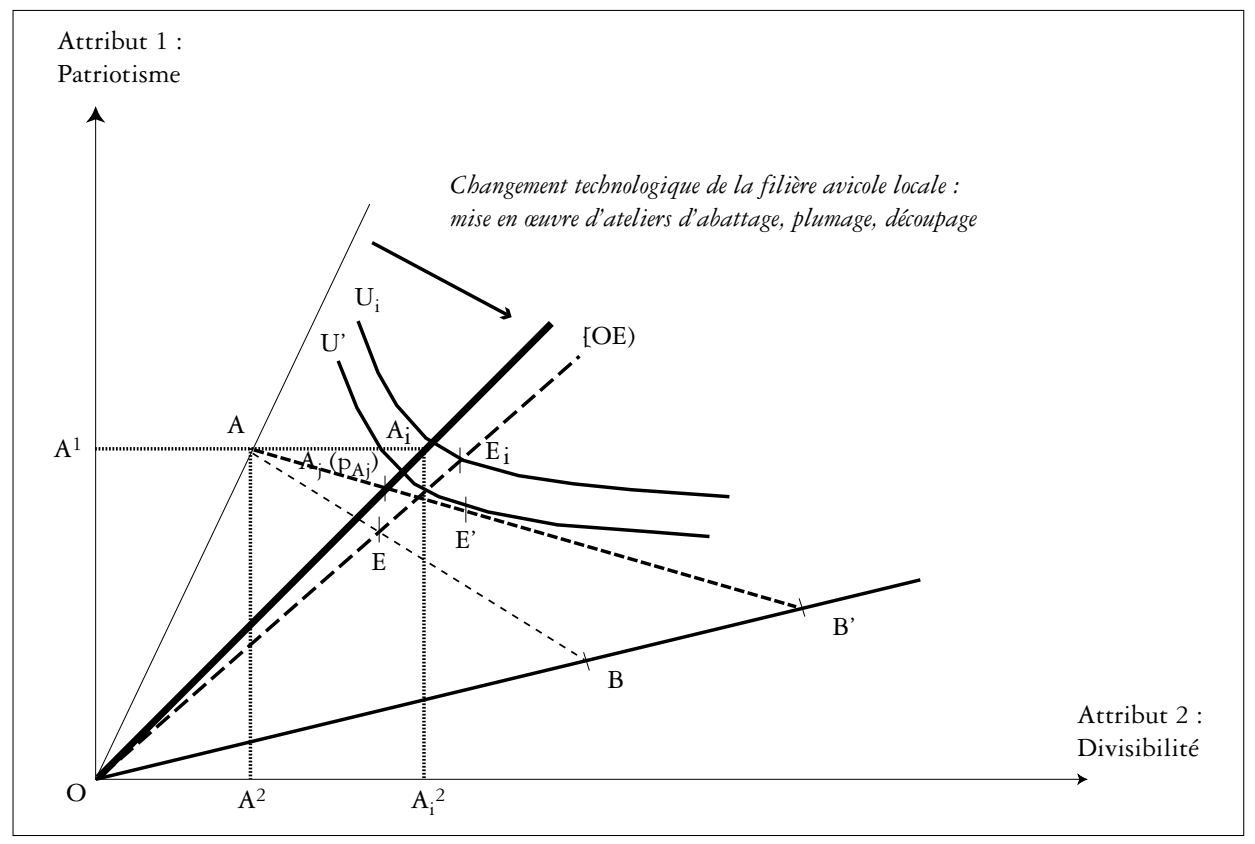


s'opère sans surcroît de coût. Autrement dit, le prix du poulet A reste pA. Cette hypothèse est posée pour la compréhension de la construction du graphique. Elle sera levée dans un second temps.

Sous cette hypothèse, la demi-droite des consommations possibles se déplace de $[\mathrm{OA})$ à $\left[\left(\mathrm{OA}_{\mathrm{i}}\right)\right.$ : pour un même niveau $\mathrm{A}^{1}$ d'attribut 1 retiré de la consommation exclusive de poulet $A$, le consommateur peut profiter d'un niveau $A_{i}{ }^{2}>A^{2}$ d'attribut 2 . Dans cette situation, la nouvelle droite de budget est représentée par $\left(\mathrm{A}_{\mathrm{i}} \mathrm{B}^{\prime}\right)$. La maximisation de l'utilité sous contrainte de budget fait passer l'équilibre de $\mathrm{E}^{\prime}$ à $\mathrm{E}_{\mathrm{i}}$. Le niveau d'utilité atteint $U_{i}>U$ '. A l'équilibre $E_{j}$, le poulet local $A$ a reconquis la part du marché local perdue suite à l'ouverture aux importations du poulet B. En effet, dans le cas représenté sur le graphique $2, \mathrm{E}_{\mathrm{i}}$ étant situé sur $[\mathrm{OE}$ ), la répartition des parts de marché entre poulets $A$ et $B$ est la même que dans la situation d'un marché protégé par un droit de douane élevé (équilibre E).

Ainsi, le développement d'une filière de transformation des poulets locaux adaptée aux attentes des consommateurs en terme de divisibilité du produit, et sans répercussion sur le prix du poulet local, permettrait d'accroittre la consommation de poulet local au détriment des découpes de poulet importées. Toutefois, le développement d'une telle filière d'abattage, plumage et découpe de poulet entraînerait nécessairement des coûts de transformation inévitablement répercutés sur le prix à la consommation pA, qui s'ajustera à la hausse. La vraie question est de déterminer la marge de manœuvre pour les acteurs de la filière : à partir de quel prix $\mathrm{p}_{A j}$ la mise en place d'une filière de transformation n'entraîne-t-elle plus de gain de parts de marché du poulet local au détriment du poulet importé congelé par rapport à la situation d'équilibre E' ?

Cette question peut se résoudre graphiquement. Toute augmentation du prix à la consommation va graphiquement se traduire par un déplacement du point $A_{i}$ le long de la demi-droite $\left[\mathrm{OA}_{\mathrm{i}}\right)$ en direction de l'origine $\mathrm{O}$. Le prix $\mathrm{p}_{\mathrm{Aj}}>\mathrm{p}_{\mathrm{A}}$ correspond au point $A_{j}$ situé à l'intersection de la demi-droite $\left[\mathrm{OA}_{\mathrm{i}}\right)$ et de la droite des budgets $(\mathrm{AB}$ ') . A ce prix, on retrouve l'équilibre E' et le niveau d'utilité U'. Pour tout prix supérieur à $\mathrm{p}_{\mathrm{Aj}}$, l'utilité par rapport à la situation initiale se détériore davantage.

Afin de mesurer cette marge de manœuvre, il serait intéressant de compléter cette approche graphique en déterminant la valeur consentie par les consommateurs camerounais aux attributs de consommation des poulets, en particulier celui de la divisibilité. Une telle mesure de la part du prix du poulet affectée à l'attribut divisibilité serait à mettre en regard du surcoût de prix imposé par la mise en œuvre d'une filière d'abattage, plumage et découpe, et renseignerait sur l'opportunité de se lancer dans cette filière.

\subsection{Les effets d'une campagne de mobilisation à l'encontre des importations de poulet}

Dans quelle mesure une campagne de mobilisation peut-elle jouer en faveur d'une reconquête, par le poulet local, du marché de la consommation de poulet au Cameroun? Quels peuvent être les effets d'une campagne telle que celle menée par exemple par Agir Ici depuis 2004, et reprise par de nombreux médias nationaux et internationaux? 
Cette campagne vise à alerter l'opinion publique internationale et faire pression sur les pays exportateurs de poulet vers l'Afrique des méfaits de ces exportations pour les économies locales africaines. Les exportations de poulet sont d'autant plus stigmatisées qu'elles bénéficient bien souvent d'aide à la filière avicole, ce qui leur permet d'être artificiellement très compétitives sur le marché mondial. Cette campagne véhicule également d'autres messages, hors du registre de la critique des politiques commerciales, qui sont relayés par la presse nationale camerounaise et internationale. Ainsi, par exemple, l'argumentaire en faveur de la sauvegarde de la filière avicole en Afrique ne fait pas la différence entre le poulet de chair, issu d'élevages rationalisés, qui est effectivement directement concurrencé par les importations de découpes congelées, et le poulet villageois qui reste au contraire relativement préservé de cette concurrence $^{3}$. Cette simplification permet de stigmatiser les effets négatifs des importations sur l'aviculture locale, laissant aux lecteurs le soin d'imaginer de quelle agriculture il s'agit. Enfin elle met en évidence les défaillances de la chaîne de froid des découpes congelées de poulet importées, depuis leur importation jusquà leur consommation. Autrement dit, à l'attribut "patriotisme", la campagne associe conjointement la « qualité sanitaire », laissant entendre que ces deux attributs sont liés.

Les effets d'une telle campagne peuvent s'analyser à deux stades.

(i) Si cette campagne obtient gain de cause, c'est-à-dire l'abolition des subventions aux exportations et/ou le rétablissement d'un droit de douane à l'importation, suffisants pour que le prix du poulet $B$ retrouve son niveau initial $\mathrm{p}_{\mathrm{B}}$, alors le résultat de cette campagne est illustré, sur le graphique 1, par un retour à l'équilibre $\mathrm{E}$.

(ii) En attendant une telle issue, notre cadre d'analyse nous permet d'illustrer les effets de la campagne en cours, dès lors qu'elle est largement relayée, auprès des consommateurs, par les médias camerounais. C'est ce qui est représenté sur le graphique 3.

On part à nouveau de la situation d'équilibre E' obtenue après ouverture aux importations de découpes de poulet congelées. Le niveau d'utilité atteint est U'; la droite de budget passe par [AB'].

La campagne met l'accent sur les méfaits des importations de poulet pour l'économie locale. Ce faisant, elle peut, si elle est suffisamment relayée par les médias camerounais, abaisser la perception, par les consommateurs, de l'attribut de consommation $1 \mathrm{du}$ poulet congelé, voire y corréler la perception d'un niveau abaissé de « qualité sanitaire » du poulet B, jouant exactement dans le même sens.

Sous ces hypothèses, la demi-droite des consommations possibles se déplace de [OB') à $\left[\mathrm{OB}^{*}\right)$ : pour un même niveau $\mathrm{B}^{2}$ ' d'attribut 2 retiré de la consommation exclusive de poulet $\mathrm{B}$, le consommateur peut profiter d'un niveau $\mathrm{B}^{1 *}<\mathrm{B}^{1}$, d'attribut 2. La droite de budget passe donc maintenant par le segment [AB*]. Dans le cas représenté sur le graphique 3, la campagne de sensibilisation est suffisamment forte pour réduire le niveau d'attribut 1 du produit $\mathrm{B}$, perçu par les consommateurs, au

\footnotetext{
3 Voir, par exemple, Ouest France, septembre 2006.
} 
Graphique 3. Effets d'une campagne de mobilisation en faveur de la consommation locale de poulet

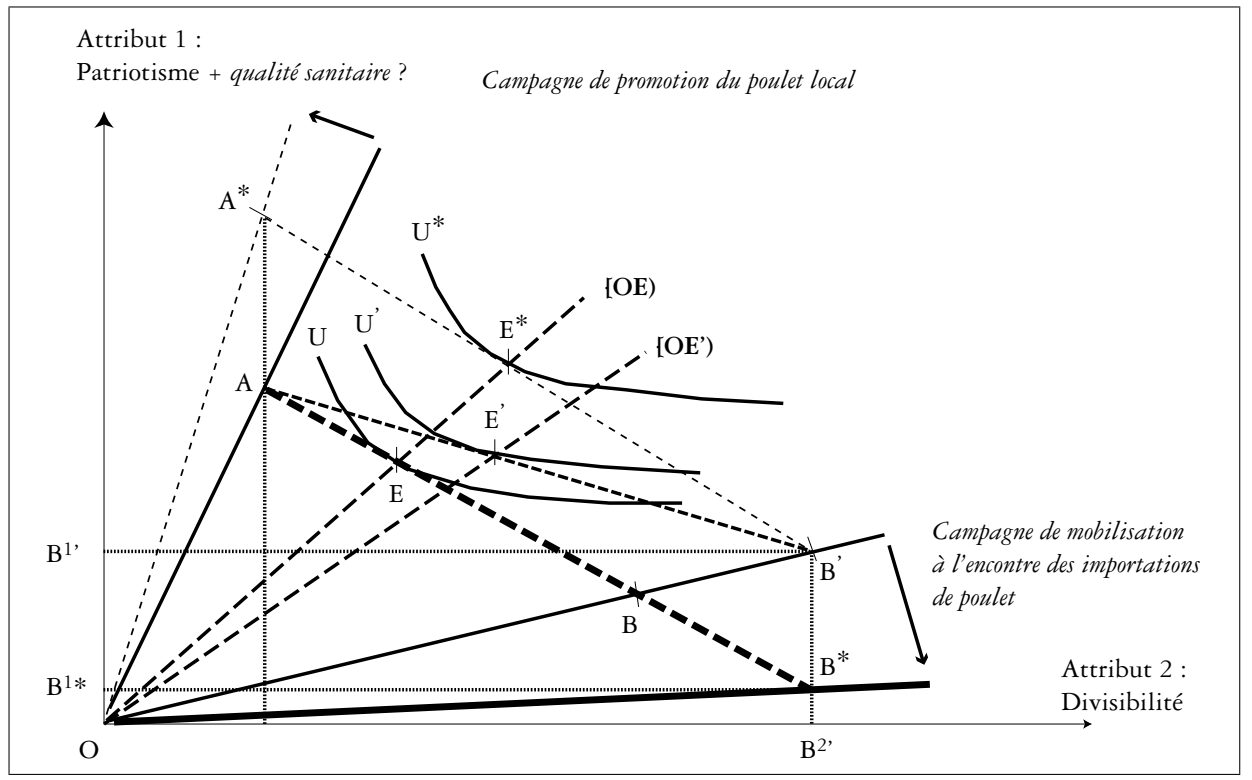

niveau $\mathrm{B}^{1 *}$. Le segment $\left[\mathrm{AB}^{*}\right]$ est alors confondu avec la droite $(\mathrm{AB})$, droite des budgets avant réduction des droits de douane sur les importations. La maximisation de l'utilité sous contrainte de revenu permet de revenir au niveau d'iso-utilité $U$ (avec $\mathrm{U}<\mathrm{U}$ '), au point d'équilibre $\mathrm{E}$, correspondant à la situation avant diminution des droits de douane. On constate que le poulet local A a reconquis la part du marché local concédée suite à l'ouverture aux importations du poulet $\mathrm{B}$. Une campagne moins forte amènerait à une situation intermédiaire entre l'équilibre $\mathrm{E}$ et l'équilibre $\mathrm{E}$ ', en termes de niveaux d'utilité et de répartition du marché entre $\mathrm{A}$ et $\mathrm{B}$. Notons que dans tous les cas, une telle campagne a pour effet de réduire le niveau d'utilité retiré par les consommateurs de leur consommation de poulet.

Il est possible, par un raisonnement similaire, d'évaluer la nature des effets d'une campagne de promotion du poulet local, au nom du patriotisme pour le poulet A au moyen du graphique 3. Si cette campagne était d'ampleur assez grande (passage de A en $A^{*}$ ), elle pourrait également déboucher sur une reconquête des parts de marché perdues par la réduction du droit de douane sur les importations (nouvel équilibre $\mathrm{E}^{*}$ ). A la différence de la campagne de mobilisation à l'encontre des poulets importés, une campagne de promotion des poulets locaux s'accompagne d'un gain d'utilité pour les consommateurs, qui se traduit graphiquement par une courbe d'iso-utilité atteinte supérieure $\left(\mathrm{U}^{*}>\mathrm{U}^{\prime}\right)$.

Comme dans la section précédente, une évaluation de la valeur affectée par les consommateurs aux attributs de consommation du poulet (patriotisme, qualité sanitaire...), dont la perception peut être modifiée par une campagne de sensibilisation, serait essentielle pour compléter notre approche. Elle permettrait par exemple de 
déterminer le niveau de protection minimal requis pour permettre au poulet de chair local, compte tenu de ses attributs, de rester sur le marché malgré la concurrence des découpes congelées.

\section{Conclusions et perspectives}

Cet article présente une application de la théorie de Lancaster à la consommation de poulet en Afrique, dans une situation où la production avicole locale est concurrencée par des produits importés, et où l'objectif de reconquête du marché domestique par le poulet local est recherché. La situation décrite ici, analysée au moyen d'un modèle d'équilibre partiel supposant l'homogénéité des produits locaux et importés, conclurait classiquement à la nécessité, pour maintenir la filière de production locale, de remettre en place une protection douanière à l'encontre des importations de poulet versus à l'accroissement nécessaire de la compétitivité prix des productions locales. Cet article permet de montrer que la prise en compte d'une certaine forme de différenciation des produits vient compléter le cadre d'analyse classique. Ainsi, la prise en compte des attributs de consommation déterminant les choix des consommateurs, mise en évidence par une enquête auprès d'un échantillon représentatif de consommateurs camerounais, permet de formaliser, au moins sous forme graphique, l'intérêt (et les limites) de scénarios alternatifs ou concomitants.

Le premier scénario serait la mise en oeuvre d'une filière de découpe rendant le poulet de chair local divisible, comme le sont déjà les découpes de poulet importées. Le poulet de chair local cumulerait alors des niveaux élevés des attributs patriotisme et divisibilité, recherchés par les consommateurs. Le second serait la mise en place d'une campagne de mobilisation, doublée d'information auprès des consommateurs locaux, qui viendrait modifier la perception des attributs des différents produits par les consommateurs. Ainsi, nous montrons qu'une campagne de promotion des attributs propres au poulet local serait plus favorable aux consommateurs qu'une campagne de dénigrement des poulets importés. Pour être effectives, des campagnes de ce type doivent être accompagnées, sur les lieux de vente et de consommation, d'une information aux consommateurs quant à l'origine du poulet offert. Sur les grands marchés urbains, une information officielle sur l'origine nécessite d'être divulguée par un organisme digne de confiance. Si l'intervention de l'Etat est alors nécessaire, la crédibilité de la démarche de certification aux yeux des consommateurs sera renforcée si les professionnels de la filière y sont associés (Cheyns et Bricas, 2003). Toutefois dans les restaurants, l'effet sur les consommateurs d'une simple pancarte du type "Ici le poulet servi est du poulet camerounais » mériterait d'être évalué, compte tenu de l'importance des relations inter-personnelles dans le lien de confiance entre client et commerçant (Cheyns et Bricas, 2003).

Pour les deux scénarii, la mise en œuvre des stratégies envisagées n'est cependant possible qu'à la condition que la valorisation du produit local induite ne détériore pas sa position concurrentielle. S'en assurer passe par une comparaison du consentement marginal à payer des consommateurs pour tel ou tel attribut au coût de l'accroissement marginal de la perception de cet attribut par le consommateur, obtenu par la mise en œuvre de la stratégie préconisée. Les résultats de cet examen détermineront 
l'orientation de la politique industrielle du secteur et le niveau de protection aux frontières qui doit éventuellement l'accompagner. A ce stade, il est donc nécessaire de compléter notre analyse par la mesure du consentement à payer des consommateurs pour tel ou tel attribut, à l'exemple d'autres travaux réalisés pour d'autres produits, évoqués au début de la section 2. C'est dans cette logique que dans le cadre de travaux de recherche en cours, les données d'enquêtes réalisées à Yaoundé sont utilisées pour développer une approche hédonique du consentement à payer des consommateurs camerounais pour les différents types de poulet présents sur les marchés urbains.

\section{Bibliographie}

ACDIC (Association citoyenne pour la défense des intérêts des consommateurs) (2005) L'importation massive de poulet congelé au Cameroun : état des lieux, enjeux et alternatives, Rapport d'études, 168 p.

ACDIC (Association citoyenne pour la défense des intérêts des consommateurs) (2004) Etat des lieux de la filière avicole au Cameroun, Rapport d'études, 32 p.

Awono Bessa C., Laroche Dupraz C., Grongnet J.-F., Vermersch D., Havard M. et Lhuissier A. (2005) Déterminants de la consommation urbaine de poulet de chair au Cameroun: cas de la ville de Yaoundé, Atelier «Agriculture et développement urbain en Afrique de l'Ouest et du Centre», Yaoundé, Cameroun, 30 octobre -3 novembre.

Cameroon Tribune (voir, par exemple, 05/09/2003, 20/08/2004, 03/02/2005) bttp:// www.cameroon-tribune.net/

CCIMA (Chambre de commerce et industrie des mines et de l'artisanat) (2005) Expansion du commerce intra- et inter-régional entre les pays de la CEMAC et de l'UEMOA, Étude de l'offre et de la demande sur les produits alimentaires, Rapport, Cameroun, 159 p.

Cheyns E., Bricas N. (2003) La construction de la qualité des produits alimentaires, le cas du doumbala, des céréales et des viandes sur le marché de Ouagadougou au Burkina Faso, Document de travail, CIRAD, 82 p.

Coestier B., Marette S. (2004) Économie de la qualité, Paris, La Découverte, collection Repères, $121 \mathrm{p}$.

Dalton T.J. (2004) A household hedonic model of rice traits: Economic values from farmers in West Africa, Agricultural Economics 31, 149-159.

Direction générale des douanes du Cameroun, bttp://www.douanescustoms.gov.cm/, consulté en octobre 2007. 
Dury S., Meuriot V., Fliedel G., Blancher S., Bore Guindo F., Drame D., Bricas N., Diakite L. and Cruz J.F. (2007) Retail market prices of fonio reveal the demand for quality characteristics in Bamako, Mali, Paper prepared for presentation at the $106^{\text {th }}$ seminar of the EAAE, Montpellier, 25-27 October, 22 p.

INS (Institut national de la statistique) (2004) Statistiques obtenues en 2005, pouvant être commandées depuis bttp://www.statistics-cameroon.org/.

Jabbar M.A. (1998) Buyer preferences for sheep and goats in Southern Nigeria: A hedonic price analysis, Agricultural Economics 18, 21-30.

Lancaster K.J. (1966a) A new approach to consumer theory, The Journal of Political Economy 54(2), 132-157.

Lancaster K.J. (1966b) Allocation and distribution theory: Technological innovation and progress. Change and innovation in the technology of consumption, American Economic Review 56(2), 14-23.

Lançon F., David Benz H. (2007) Rice import in West Africa: Trade regimes and food policy formulation, Poster prepared for presentation at the $106^{\text {th }}$ seminar of the EAAE, Montpellier, 25-27 October.

Njonga B. (2005) L'agriculture ouest-africaine à l'épreuve de la libéralisation des échanges, Rapport de l'Association civile de défense des consommateurs, Yaoundé, Cameroun, 17 p.

Oxfam France - Agir ici (2004) Exportations de poulets : l'Europe plume l'Afrique, Paris, Campagne d'information et de mobilisation, $\mathrm{n}^{\circ} 68,4 \mathrm{p}$. (un résumé de ce document est consultable sur le site http://www.oxfamfrance.org)

Sanogo D., Masters A. (2002) A market-based approach to child nutrition: Mothers' demand for quality certification of infant foods in Bamako, Mali, Food Policy 27(3), 251-268.

Teleu Ngandeu E., Ngatchou A. (2006) Première évaluation du secteur avicole au Cameroun : structure et importance du secteur avicole commercial et familial pour une meilleure compréhension de l'enjeu de l'Influenza aviaire, Rapport des consultants nationaux, Organisation des Nations Unies pour l'alimentation et l'agriculture, FAO, Projet OSRO/GLO/MUL. 